\title{
Simulations of the adsorption of ionic species at polarisable liquid|liquid interfaces
}

\author{
Bin Su, Nicolas Eugster, Hubert H. Girault * \\ Laboratoire delectrochimie Physique et Analytique, Institut de Chimie Moleculaire et Biologique, Ecole Polytechnique Fédérale de Lausanne, \\ CH-1015 Lausanne, Switzerland
}

Received 5 August 2004; received in revised form 8 November 2004; accepted 12 November 2004

Available online 30 December 2004

\begin{abstract}
The adsorption of ions at the interface between two immiscible electrolyte solutions (ITIES) is primarily controlled by the potential distribution across the interface, which in turn is influenced by the adsorption process. In the present paper, we simulate the effect of the adsorption of charged species on the charge distribution at the ITIES based on the classical description of the interface employing the Gouy-Chapman model. The inner layer is considered as a charged plane, where the ionic adsorption takes place. The potential at this plane is determined by the electro-neutrality condition. Various adsorption isotherms are considered, including potential dependent isotherms based on the Langmuir and Frumkin adsorption models. The potential distribution and the charge density profile are derived by solving the Poisson-Boltzman equation numerically. We show that the charge distribution in the interfacial region is significantly affected by the adsorption of ionic species. Under certain conditions, the adsorption results in a nonmonotonic potential distribution with a potential trap at the interface.
\end{abstract}

(C) 2004 Elsevier B.V. All rights reserved.

Keywords: Adsorption; Charged species; ITIES; Potential distribution; Charge density; Differential capacitance; Gouy-Chapman model

\section{Introduction}

The molecular structure of the interface between two immiscible electrolyte solutions (ITIES) remains a rather controversial and interesting topic. Knowledge of the interfacial structure is a prerequisite for understanding a variety of interfacial phenomena, such as charge transfer across the interface, adsorption and orientation of ionic and neutral species at the interface [1-3]. Early models stemming from classical electrochemical and thermodynamic analyses [4-15], such as differential capacitance and surface tension measurements, have depicted the interface as two space charge regions separated by an inner layer of solvent mole-

\footnotetext{
${ }^{*}$ Corresponding author. Tel.: +4121693 3145/3151; fax: +4121 6933667.

E-mail address: hubert.girault@epfl.ch (H.H. Girault).
}

cules. This picture, referred to as the modified Vervey-Niessen model (MVN) [4], has been widely used although there have been some controversies on the nature of the inner layer $[14,16]$. Indeed, it has been proposed that the inner layer consists of a mixed solvent layer, resulting in the overlap of the two adjacent diffuse layers $[17,18]$.

During the past decade, much effort has been devoted to detailed investigations of the structure of the liquid|liquid interface, employing spectroscopic and optical techniques as well as computer simulations. Molecular dynamics and Monte-Carlo computations [19-22] suggest that on the picosecond timescale the interface is molecularly sharp, with capillary waves extending over $1 \mathrm{~nm}$. Thus, averaging the solvent distribution over several ps defines a region of about $1 \mathrm{~nm}$ thick in which the solvent densities change monotonically with the coordinate perpendicular to the interface. 
However, molecular dynamics simulations mainly focus on the neat molecular interface with only a few ions being considered. Computer simulation has been extended to more complex systems by the use of the lattice gas model, which is a rather useful theoretical approach to model the space charge regions at the interface [2327]. The use of this model allowed the computation of charge transfer, specific ion adsorption, and ion pairing processes, as well as of the interfacial structure. In addition, the modelisation of the differential capacitance of the ITIES has been carried out using theoretical models based on ionic association [28,29], ionic penetration [30], and capillary waves [31]. Experimentally, the interfacial width and roughness of the liquid|liquid interface have been addressed by X-ray reflectivity [32-34] and neutron reflection measurements [33,35]. The results obtained with these techniques appear to confirm the predictions of computer simulations. Moreover, studies using vibrational sum-frequency spectroscopy (VSFS) have demonstrated that the water|DCE interface is molecularly disordered with properties similar to a mixed solvent interfacial region [18]. In addition, the characteristic frequencies of the capillary waves induced at the ITIES by thermal fluctuations have been measured by quasi-elastic laser scattering (QELS) [36]. The interface has also been probed by other interfacially sensitive spectroscopic and optical techniques under total internal reflection geometries, including absorption [37], fluorescence [38,39], resonance Raman [40] spectroscopies and surface second harmonic generation (SSHG) [41]. These techniques have provided important insight into the structure of the interface and the adsorption and molecular orientation of species at the interface, as well as the interfacial charge transfer reactions.

Over the years, much effort has been dedicated to ion adsorption and organisation at the ITIES. The mechanism of these processes is primarily controlled by the molecular structure of the interface, which in turn determines the potential profile across the interface. However, although the molecular adsorption at the interface alters the interfacial structure, many analyses do not take into account the change of the electrical potential profile. Theoretical developments allowing the unambiguous interpretation of the effect of ionic adsorption on the interfacial structure have not been reported yet. In the present work, we propose to consider various macroscopic models of the adsorption of ionic species from the aqueous phase to the interface to simulate the potential profile, charge density and differential capacitance at the ITIES. The gist of the calculation is the definition of a charged plane and two diffuse layers linked by the electro-neutrality condition. We show here that the interfacial composition is significantly altered by the adsorption of ionic species, resulting in a substantial change in the potential distribution across the interface.

\section{Theory and mathematical descriptions}

\subsection{Microscopic model of the interface structure}

The microscopic model of the interfacial composition is depicted schematically in Fig. 1. An inner layer separates two diffuse layers, which are classically described by the Gouy-Chapman (GC) theory. The total potential difference splits into three parts

$$
\begin{aligned}
\Delta_{\mathrm{o}}^{\mathrm{w}} \phi & =\left(\phi^{\mathrm{w}}-\phi^{2}\right)+\left(\phi^{2}-\phi^{1}\right)+\left(\phi^{1}-\phi^{\mathrm{o}}\right) \\
& =\Delta_{2}^{\mathrm{w}} \phi+\Delta_{1}^{2} \phi+\Delta_{\mathrm{o}}^{1} \phi,
\end{aligned}
$$

where $\Delta_{1}^{2} \phi$ is the potential drop across the inner layer and $\Delta_{2}^{\mathrm{w}} \phi$ and $\Delta_{\mathrm{o}}^{1} \phi$ are the potential drops across the diffuse layers in the aqueous and organic phases, respectively.

For the sake of simplicity, we make three assumptions:

1. The potential drop across the inner layer is negligible, that is, $\Delta_{1}^{2} \phi=\phi^{2}-\phi^{1}=0$. The inner layer is reduced to a charged plane. This assumption is compatible with the GC model that considers ions as point charges. Therefore, the total potential drop takes place over two diffuse layers

$\Delta_{\mathrm{o}}^{\mathrm{w}} \phi=\Delta_{2}^{\mathrm{w}} \phi+\Delta_{\mathrm{o}}^{2} \phi$.

2. We consider only the adsorption from the aqueous phase, which is supposed to take place at the charged plane. Furthermore, we assume that the adsorption of ionic species does not change the dielectric environment of the interface and the GC theory is still considered to be valid in the presence of adsorption. Given that the supporting electrolyte is in large excess of the adsorbate, the charge density due to the adsorption of ionic species and that due to the electrolytes in the diffuse layer are additive.

3. In actual calculations, it is taken that $\phi^{\mathrm{w}}=0$, and $x_{1}=x_{2}=0$. The adsorption takes place on the geometric surface dividing the two phases.

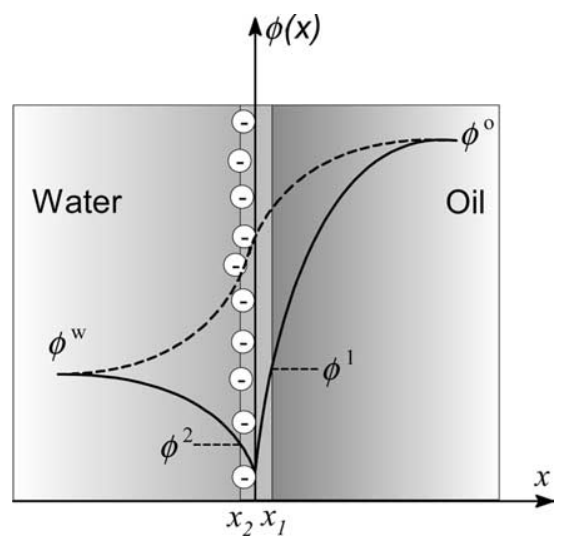

Fig. 1. Schematic representation of the potential profiles across the ITIES in the absence (dash line) and presence (solid line) of specific adsorption of anionic species from the aqueous phase to the interface. 


\subsection{Potential distribution}

For clarity, we shall present here again the classical GC theory [42] and its application to ITIES. Assuming that the charge density is uniform in a volume element parallel to the interface, the Poisson equation describes the relation between the potential gradient and the charge density. In the case of a 1:1 supporting electrolyte, this relation becomes

$\frac{\partial^{2} \phi(x)}{\partial x^{2}}=\frac{2 F c^{\mathrm{r}}}{\varepsilon_{0} \varepsilon^{\mathrm{r}}} \sinh \left\{\frac{F\left[\phi(x)-\phi^{\mathrm{r}}\right]}{R T}\right\}$,

where $\varepsilon_{0}$ is the vacuum permittivity, and $F, R, T$ are, respectively, the Faraday constant, the gas constant and the temperature. Also, $c^{\mathrm{r}}, \varepsilon^{\mathrm{r}}$, and $\phi^{\mathrm{r}}$ are the bulk concentration of the supporting electrolyte, the relative permittivity, and the bulk potential in the appropriate phase $(\mathrm{r}=\mathrm{w}$ or $\mathrm{o})$, respectively.

We integrate Eq. (3) with the following boundary conditions:

$x \rightarrow-\infty, \phi(x) \rightarrow \phi^{\mathrm{w}} \quad$ and $\quad \frac{\partial \phi(x)}{\partial x} \rightarrow 0$,

$x \rightarrow \infty, \phi(x) \rightarrow \phi^{\mathrm{o}} \quad$ and $\quad \frac{\partial \phi(x)}{\partial x} \rightarrow 0$

to obtain the potential gradients in the two diffuse layers:

$\left.\frac{\partial \phi(x)}{\partial x}\right|_{-\infty<x \leqslant x_{2}}=\sqrt{\frac{8 R T c^{\mathrm{w}}}{\varepsilon_{0} \varepsilon^{\mathrm{w}}}} \sinh \left[f\left(\phi(x)-\phi^{\mathrm{w}}\right)\right]$,

$\left.\frac{\partial \phi(x)}{\partial x}\right|_{x_{1} \leqslant x<\infty}=-\sqrt{\frac{8 R T c^{\mathrm{o}}}{\varepsilon_{0} \varepsilon^{\mathrm{o}}}} \sinh \left[f\left(\phi(x)-\phi^{\mathrm{o}}\right)\right]$,

where $f$ is equal to $F / 2 R T$.

Integration of Eqs. (4a) and (4b) with the additional boundary condition

$x=x_{1}=x_{2}, \quad \phi(x)=\phi^{2}$

gives the potential distributions in two diffuse layers:

$$
\begin{aligned}
\left.\phi(x)\right|_{-\infty<x \leqslant x_{2}}= & \phi^{\mathrm{w}}+\frac{2}{f} \operatorname{arctanh} \\
& \times\left\{\exp \left[\kappa^{\mathrm{w}}\left(x-x_{2}\right)\right] \tanh \left[\frac{f}{2}\left(\phi^{2}-\phi^{\mathrm{w}}\right)\right]\right\},
\end{aligned}
$$

$$
\begin{aligned}
\left.\phi(x)\right|_{x_{1} \leqslant x<\infty}= & \phi^{\mathrm{o}}+\frac{2}{f} \operatorname{arctanh} \\
& \times\left\{\exp \left[\kappa^{\mathrm{o}}\left(x-x_{1}\right)\right] \tanh \left[\frac{f}{2}\left(\phi^{2}-\phi^{\mathrm{o}}\right)\right]\right\},
\end{aligned}
$$

where $\kappa^{\mathrm{w}}=F\left(2 c^{\mathrm{w}} / R T \varepsilon_{0} \varepsilon^{\mathrm{w}}\right)^{1 / 2}$ and $\kappa^{\mathrm{o}}=F\left(2 c^{\mathrm{o}} / R T \varepsilon_{0} \varepsilon^{\mathrm{o}}\right)^{1 / 2}$. The potential $\phi^{2}$ is defined by the electro-neutrality condition for the entire interfacial region

$\sigma^{\mathrm{w}}+\sigma^{\mathrm{o}}+\sigma^{\mathrm{ads}}=0$,

where $\sigma^{\text {ads }}$ is the surface charge density due to the adsorption of ionic species from the aqueous phase to the interface and will be specified in the different adsorption models. $\sigma^{\mathrm{w}}$ and $\sigma^{\mathrm{o}}$ are the surface charge densities in the diffuse layers of the aqueous and organic phases, respectively. They are given classically by:

$$
\begin{aligned}
& \sigma^{\mathrm{w}}=-\left.\varepsilon_{0} \varepsilon^{\mathrm{w}} \frac{\partial \phi(x)}{\partial x}\right|_{x=x_{2}}=-a \sinh \left[f\left(\phi^{2}-\phi^{\mathrm{w}}\right)\right], \\
& \sigma^{\mathrm{o}}=-\left.\varepsilon_{0} \varepsilon^{\mathrm{o}} \frac{\partial \phi(x)}{\partial x}\right|_{x=x_{1}}=-b \sinh \left[f\left(\phi^{2}-\phi^{\mathrm{o}}\right)\right],
\end{aligned}
$$

where $a=\sqrt{8 R T \varepsilon_{0} \varepsilon^{\mathrm{w}} c^{\mathrm{w}}}$ and $b=\sqrt{8 R T \varepsilon_{0} \varepsilon^{\mathrm{o}} c^{\mathrm{o}}}$.

\subsection{Charge density and differential capacitance}

According to Eq. (6) we define the interfacial charge density as

$\sigma=\sigma^{\mathrm{w}}+\sigma^{\mathrm{ads}}=-\sigma^{\mathrm{o}}$.

The interfacial capacitance is given as the derivative of the charge density with respect to the Galvani potential difference [28]

$\frac{1}{C_{\mathrm{d}}}=\frac{\mathrm{d} \Delta_{\mathrm{o}}^{\mathrm{w}} \phi}{\mathrm{d} \sigma}=\frac{\mathrm{d} \Delta_{2}^{\mathrm{w}} \phi}{\mathrm{d} \sigma}+\frac{\mathrm{d} \Delta_{\mathrm{o}}^{2} \phi}{\mathrm{d} \sigma}=\frac{1}{C^{\mathrm{w}}}+\frac{1}{C^{\mathrm{o}}}$.

The total capacitance can be represented as two capacitances in series. The individual capacitances are given as follows:

$C^{\mathrm{w}}=\frac{\mathrm{d} \sigma}{\mathrm{d} \Delta_{2}^{\mathrm{w}} \phi}=\frac{\mathrm{d}\left(\sigma^{\mathrm{w}}+\sigma^{\mathrm{ads}}\right)}{\mathrm{d}\left(\phi^{\mathrm{w}}-\phi^{2}\right)}=-\frac{\mathrm{d}\left(\sigma^{\mathrm{w}}+\sigma^{\mathrm{ads}}\right)}{\mathrm{d}\left(\phi^{2}-\phi^{\mathrm{w}}\right)}$,

$C^{\mathrm{o}}=\frac{\mathrm{d} \sigma}{\mathrm{d} \Delta_{\mathrm{o}}^{2} \phi}=-\frac{\mathrm{d} \sigma^{\mathrm{o}}}{\mathrm{d}\left(\phi^{2}-\phi^{\mathrm{o}}\right)}$.

\section{Results and discussions}

All the results presented in this paper were obtained using the Maple 9.0 (Maplesoft, Waterloo Maple Inc.) program on a personal computer. In all simulations, the concentrations of supporting electrolytes in each phase were $c^{\mathrm{w}}=c^{\mathrm{o}}=10 \mathrm{~mol} \mathrm{~m}^{-3}$, and the relative dielectric constants were $\varepsilon^{\mathrm{w}}=70$ and $\varepsilon^{\mathrm{o}}=10$. The absolute temperature was taken as $298 \mathrm{~K}$. The Maple programs used in this paper are available in the supporting information. 


\subsection{In the absence of adsorption}

To allow further comparison, we present first the classical way to calculate the Gouy Chapman capacitance. In the absence of adsorption, that is, $\sigma^{\text {ads }}=0$, the expression of $\phi^{2}$ is obtained from Eqs. (6), (7a) and $(7 b)$

$$
\begin{aligned}
\phi^{2}= & \phi^{\mathrm{w}}+\frac{1}{f} \ln \left\{\sqrt{\exp \left(f \Delta_{\mathrm{o}}^{\mathrm{w}} \phi\right)}\right\}+\frac{1}{f} \\
& \times \ln \left\{a+b \exp \left(f \Delta_{\mathrm{o}}^{\mathrm{w}} \phi\right)\right\}+\frac{1}{f} \\
& \times \ln \left\{a \exp \left(f \Delta_{\mathrm{o}}^{\mathrm{w}} \phi\right)+b\right\}-\frac{1}{f} \\
& \times \ln \left\{a \exp \left(f \Delta_{\mathrm{o}}^{\mathrm{w}} \phi\right)+b\left[\exp \left(f \Delta_{\mathrm{o}}^{\mathrm{w}} \phi\right)\right]^{2}\right\} .
\end{aligned}
$$

By substituting Eq. (11) into Eqs. (5a) and (5b) separately, the potential distributions in the aqueous and organic diffuse layers become:

$$
\begin{aligned}
\left.\phi(x)\right|_{-\infty<x \leqslant x_{2}}= & \phi^{\mathrm{w}}+\frac{2}{f} \operatorname{arctanh} \\
& \times\left\{\exp \left[\kappa^{\mathrm{w}}\left(x-x_{2}\right)\right] \tanh \right. \\
& \left.\times\left\{\frac{1}{4} \ln \left[\frac{a+b \exp \left(-f \Delta_{\mathrm{o}}^{\mathrm{w}} \phi\right)}{a \exp \left(-f \Delta_{\mathrm{o}}^{\mathrm{w}} \phi\right)+b}\right]-\frac{f}{4} \Delta_{\mathrm{o}}^{\mathrm{w}} \phi\right\}\right\},
\end{aligned}
$$

$$
\begin{aligned}
\left.\phi(x)\right|_{x_{1} \leqslant x<\infty}= & \phi^{\mathrm{o}}-\frac{2}{f} \operatorname{arctanh} \\
& \times\left\{\exp \left[-\kappa^{\mathrm{o}}\left(x-x_{1}\right)\right] \tanh \right. \\
& \left.\times\left\{-\frac{1}{4} \ln \left[\frac{a+b \exp \left(f \Delta_{\mathrm{o}}^{\mathrm{w}} \phi\right)}{a+b \exp \left(f \Delta_{\mathrm{o}}^{\mathrm{w}} \phi\right)}\right]-\frac{f}{4} \Delta_{\mathrm{o}}^{\mathrm{w}} \phi\right\}\right\} .
\end{aligned}
$$

Inserting Eq. (11) into Eq. (8), we obtain the charge density at the interface

$$
\sigma=\frac{a b\left\{-1+\left[\exp \left(f \Delta_{\mathrm{o}}^{\mathrm{w}} \phi\right)\right]\right\}}{2 \sqrt{\exp \left(f \Delta_{\mathrm{o}}^{\mathrm{w}} \phi\right)\left[a+b \exp \left(f \Delta_{\mathrm{o}}^{\mathrm{w}} \phi\right)\right]\left[a \exp \left(f \Delta_{\mathrm{o}}^{\mathrm{w}} \phi\right)+b\right]}} .
$$

Then, from Eqs. (10a), (10b) and (13), we have:

$$
\begin{aligned}
C^{\mathrm{w}}= & -a f \\
& \times \cosh \left\{\ln \sqrt{\frac{\exp \left(-f \Delta_{\mathrm{o}}^{\mathrm{w}} \phi\right)\left[a+b \exp \left(-f \Delta_{\mathrm{o}}^{\mathrm{w}} \phi\right)\right]}{a \exp \left(-f \Delta_{\mathrm{o}}^{\mathrm{w}} \phi\right)}}\right\},
\end{aligned}
$$

$$
\begin{aligned}
C^{\mathrm{o}}= & -b f \cosh \\
& \times\left\{\ln \sqrt{\frac{\exp \left(-f \Delta_{\mathrm{o}}^{\mathrm{w}} \phi\right)\left[a+b \exp \left(-f \Delta_{\mathrm{o}}^{\mathrm{w}} \phi\right)\right]}{a \exp \left(-f \Delta_{\mathrm{o}}^{\mathrm{w}} \phi\right)}}+f \Delta_{\mathrm{o}}^{\mathrm{w}} \phi\right\} .
\end{aligned}
$$

From Eqs. (14a) and (14b) we can calculate the total differential capacitance, $C_{\mathrm{d}}$, according to Eq. (9).

The potential at $x=0$ is $\phi^{2}$, which, under the definition in this paper has a sign opposite to $\Delta_{\mathrm{o}}^{\mathrm{w}} \phi$ in the absence of adsorption. The dotted line in Fig. 2(a) shows the evolution of $\phi^{2}$ as a function of $\Delta_{\mathrm{o}}^{\mathrm{w}} \phi\left(\phi^{2}-\phi^{\mathrm{w}}=\phi^{2}\right.$ as $\left.\phi^{\mathrm{w}}=0\right)$, as calculated from Eq. (11). The parameter $\phi^{2}$ determines the magnitudes of the potential drops in the two diffuse layers, $\phi^{2}-\phi^{\mathrm{w}}$ and $\phi^{\mathrm{o}}-\phi^{2}$. They are also compared in Fig. 2(a). The larger magnitude of the potential drop in the organic phase is related to the smaller dielectric constant.

Fig. 2(b) shows the potential profiles at various potential differences. Under the conditions employed, the potential extends to within $10-20 \mathrm{~nm}$ of both sides of the interface. Fig. 3 displays the charge density and differential capacitance in the absence of adsorption at the interface. The features observed are associated with the distribution of the supporting electrolytes in the diffuse layers.

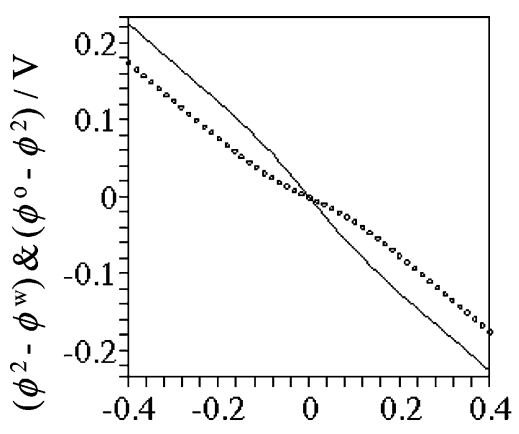

(a) $\quad \Delta_{\mathrm{o}}^{\mathrm{w}} \phi / \mathrm{V}$

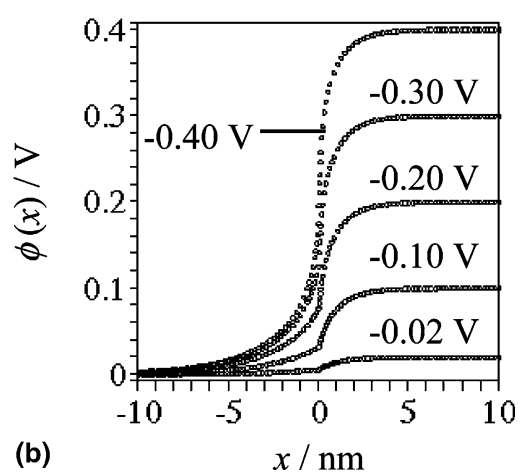

Fig. 2. (a) The magnitudes of the aqueous (point) and organic (line) diffuse layer potential drops as functions of $\Delta_{\mathrm{o}}^{\mathrm{w}} \phi$. (b) Potential profiles at various $\Delta_{\mathrm{o}}^{\mathrm{w}} \phi$ in the absence of adsorption. 

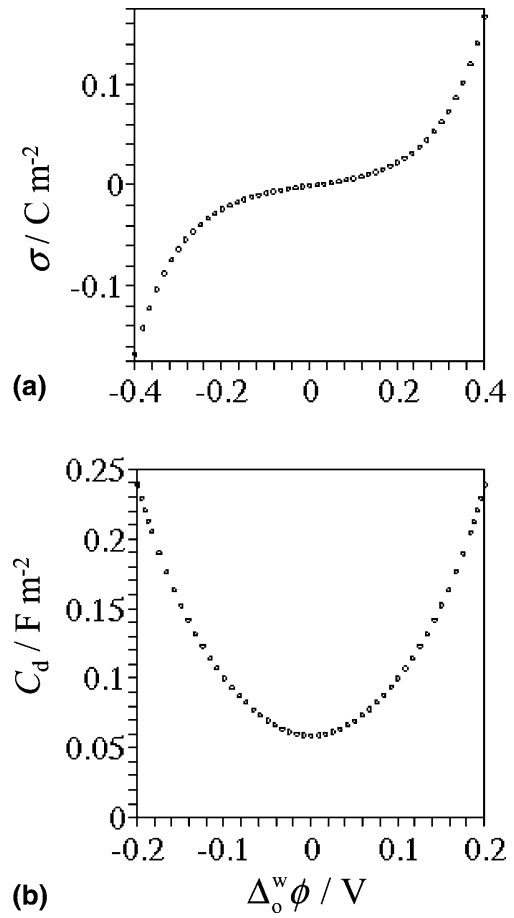

Fig. 3. Charge density (a) and differential capacitance (b) as functions of $\Delta_{\mathrm{o}}^{\mathrm{w}} \phi$ in the absence of adsorption.

\subsection{Potential independent isotherm}

We start with a simple example, potential independent isotherm, to see how the adsorption affects the potential distribution across the interface. The adsorbed charge $\sigma^{\text {ads }}$ is defined as

$\sigma^{\text {ads }}=z_{\mathrm{b}} F \Gamma^{*} \theta$,

where $z_{\mathrm{b}}$ and $\Gamma^{*}$ are the charge number and the maximum surface concentration of the absorbed species, respectively. Based on the classical description of the Langmuir isotherm, $\theta$ is the relative surface coverage, which under steady-state conditions of the adsorptiondesorption equilibrium, is given by

$\theta=\frac{\frac{a_{\mathrm{z}}}{a_{\mathrm{H}_{2} \mathrm{O}}} \exp \left(-\frac{\Delta G_{\mathrm{a}}^{0}}{R T}\right)}{1+\frac{a_{\mathrm{z}}}{a_{\mathrm{H}_{2} \mathrm{O}}} \exp \left(-\frac{\Delta G_{\mathrm{a}}^{0}}{R T}\right)}$,

where $a_{\mathrm{z}}$ is the activity of the adsorbed species in the aqueous phase and $a_{\mathrm{H}_{2} \mathrm{O}}$ the activity of water molecules in the aqueous phase. In actual calculations, concentrations, $c_{\mathrm{Z}}$ and $c_{\mathrm{H}_{2} \mathrm{O}}$ are used instead of activities for simplicity. $\Delta G_{\mathrm{a}}^{0}$ is the standard Gibbs energy of adsorption from the aqueous phase to the interface, which is independent of the potential drop in the aqueous phase. Eq. (15) is introduced in the electrostatic neutrality equation Eq. (6) to derive $\phi^{2}$.

Fig. 4(a) illustrates the effect of the adsorption of ionic species on $\phi^{2}$ for various bulk concentrations of the
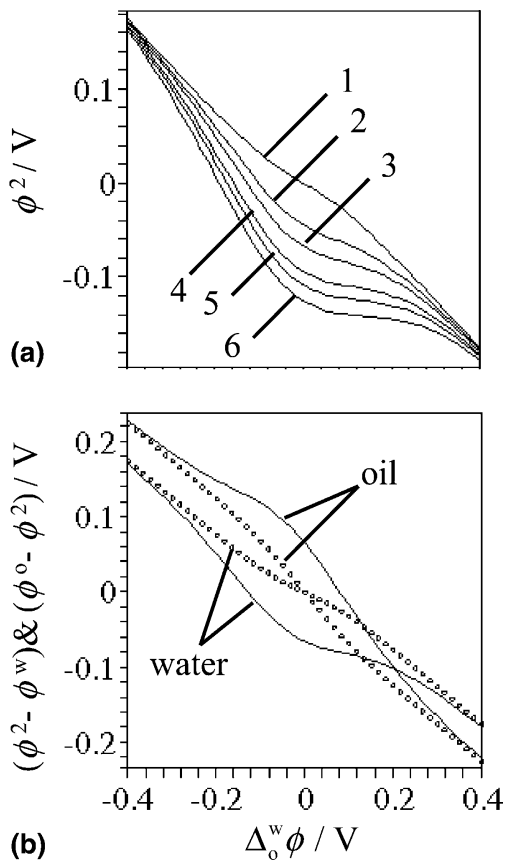

Fig. 4. (a) $\phi^{2}$ as a function of $\Delta_{\mathrm{o}}^{\mathrm{w}} \phi$ at various values of $c_{z} ; z_{\mathrm{b}}=-1$, $\Gamma^{*}=1 \times 10^{-6} \mathrm{~mol} \mathrm{~m}^{-2}, \Delta G_{\mathrm{a}}^{0}=40 \mathrm{~kJ} \mathrm{~mol}^{-1}$, and $c_{z}=0$ (1), 0.001 (2), 0.002 (3), 0.005 (4), 0.010 (5), and $0.050 \mathrm{~mol} \mathrm{~m}^{-3}$ (6). (b) The magnitudes of the diffuse layer potential drops in the absence (point) and presence (line) of adsorption when $c_{z}=0.002 \mathrm{~mol} \mathrm{~m}^{-3}$.

adsorbate. The change of $\phi^{2}$ is negligible at large potential differences, whilst much more pronounced at potential difference close to $0 \mathrm{~V}$. For example, in the case of mono-anionic species with a bulk concentration of $10^{-5} \mathrm{~mol} \mathrm{dm}^{-3}\left(\sigma^{\text {ads }}=6 \mu \mathrm{C} \mathrm{cm}^{-2}\right.$ with the parameters used in the simulation, as indicated in the figure caption), the decrease of $\phi^{2}$ induced by the adsorption is very clear in a potential range from -0.2 to $0.2 \mathrm{~V}$. As the parameter $\phi^{2}$ determines the magnitudes of the potential drops in the two diffuse layers, the changes in $\phi^{2}$ indicate the variations in the potential drops. As shown in Fig. 4(b), the magnitudes of the potential drops in the two diffuse layers are significantly altered in the low potential range. For instance, negative potential drops in the aqueous diffuse layer $\left(\phi^{2}-\phi^{\mathrm{w}}<0\right)$ are observed in a range of negative values of $\Delta_{\mathrm{o}}^{\mathrm{w}} \phi$. Under these conditions, the potential distribution is inversed as exemplified in Fig. 5 in the case of potentials of -0.10 and $-0.02 \mathrm{~V}$. This kind of potential profile has been inferred from the qualitative analysis of experimental results on the adsorption of surfactant ions at the ITIES [43]. The electric double layer composition of the interface is significantly altered under conditions where the charge density due to the adsorption exceeds that related to the supporting electrolytes in the diffuse layers.

Mathematically, Eqs. (6), (7a) and (7b) tell us that $\phi^{2}$ changes with $\sigma^{\text {ads }}$, meaning that the potential profile changes upon adsorption even though the quantity of 


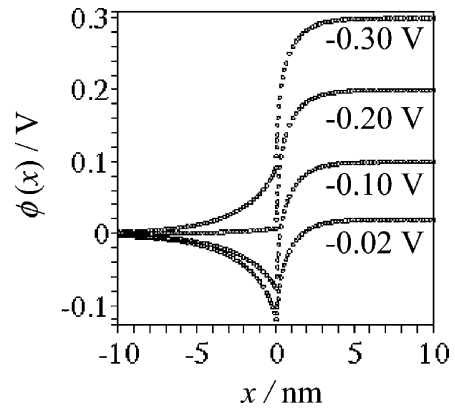

Fig. 5. Potential profiles at various $\Delta_{\mathrm{o}}^{\mathrm{w}} \phi$ in the potential independent model: $z_{\mathrm{b}}=-1 ; \quad \Gamma^{*}=1 \times 10^{-6} \mathrm{~mol} \mathrm{~m}^{-2} ; \quad \Delta G_{\mathrm{a}}^{0}=-40 \mathrm{~kJ} \mathrm{~mol}^{-1}$; $c_{z}=0.050 \mathrm{~mol} \mathrm{~m}^{-3}$.

adsorbed species remains constant. Hence, the dependence of both the interfacial charge density and differential capacitance on the potential difference is affected by the adsorption of ions, as illustrated by Fig. 6. The adsorption of anionic species manifests itself as an increase of the charge density and differential capacitance, especially at negative potentials. Furthermore, the minimum of the capacitance shifts to positive values of $\Delta_{\mathrm{o}}^{\mathrm{w}} \phi$. These features are consistent with the experimental data on the adsorption of ionic species at the ITIES [43-45].

\subsection{Langmuir isotherm}

Previous studies have suggested that the adsorption of ionic species at the ITIES is generally potential dependent [46]. The Langmuir and Frumkin isotherms
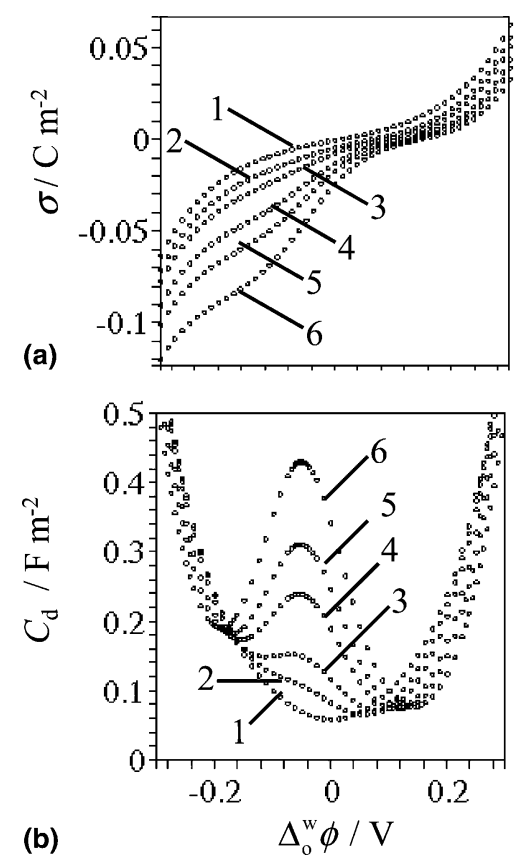

Fig. 6. Charge density (a) and differential capacitance (b) at different surface concentrations for the potential independent model. The parameters are the same as in Fig. 4(a). are most frequently used to analyze experimental data $[3,46,47]$. In the case of the Langmuir isotherm, the relative surface coverage $\theta$ is given by Higgins and Corn [48]

$$
\begin{aligned}
\theta= & \frac{a_{\mathrm{z}}}{a_{\mathrm{H}_{2} \mathrm{O}}} \\
& \times \frac{\exp \left(-\frac{\Delta G_{\mathrm{a}}^{0}}{R T}\right) \exp \left[-\frac{z_{\mathrm{b}} F}{R T}\left(\phi^{2}-\phi^{\mathrm{w}}\right)\right]}{1+\frac{a_{\mathrm{z}}}{a_{\mathrm{H}_{2} \mathrm{O}}} \exp \left(-\frac{\Delta G_{\mathrm{a}}^{0}}{R T}\right) \exp \left[-\frac{z_{\mathrm{b}} F}{R T}\left(\phi^{2}-\phi^{\mathrm{w}}\right)\right]} .
\end{aligned}
$$

All the parameters have the same meanings as in Section 3.2. The adsorbed charge $\sigma^{\text {ads }}$ can be calculated by inserting Eq. (17) into Eq. (15), which is further introduced in Eq. (6) to derive $\phi^{2}$. Although no analytical solution is obtained in this case, the symbolic expression can be evaluated numerically. This kind of treatment is extended to the computation of the potential distribution, the relative surface coverage, charge density and differential capacitance.

As from Eqs. (15) and (17), the adsorption behaviour is controlled by several variables including $z_{\mathrm{b}}, \Gamma^{*}, c_{\mathrm{z}}$, and $\Delta G_{\mathrm{a}}^{0} \cdot z_{\mathrm{b}}$ and $\Delta G_{\mathrm{a}}^{0}$ are constant for a given species. $\Gamma^{*}$ is mainly determined by the dimension of the species itself, although it may possibly be potential dependent
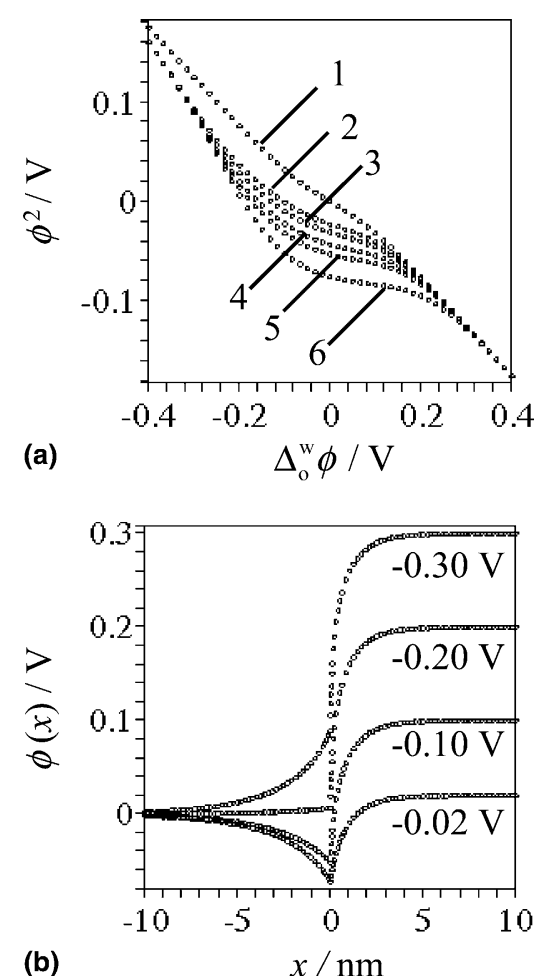

Fig. 7. (a) $\phi^{2}$ as a function of $\Delta_{\mathrm{o}}^{\mathrm{w}} \phi$ at various values of $c_{z}$. The parameters are the same as in Fig. 4(a). (b) Potential profiles at various potential differences for the Langmuir model. The parameters are the same as in Fig. 5. 
[47]. Hence, we shall not consider the variations of the parameters $z_{\mathrm{b}}, \Gamma^{*}$, and $\Delta G_{\mathrm{a}}^{0}$, but rather focus on the effect of $c_{\mathrm{z}}$, which is experimentally controllable. Fig. 7(a) shows $\phi^{2}$ as a function of the applied potential difference in the presence of various concentrations of the surfaceactive species in the aqueous phase. It is evident that $\phi^{2}$ is strongly affected by the adsorption over a large potential range. The inversion of $\phi^{2}$ from positive to negative is also observed, indicating a redistribution of the potential drop in the two diffuse layers. The corresponding potential profiles across the interface are plotted in Fig. 7(b). The results presented in Figs. 5 and 7(b) should be compared with the curves in Fig. 2(b). It is clear that the presence of adsorbed ions affects the potential distribution on both sides of the interface. This behaviour should be taken into account when reactions involving adsorbed species at liquid|liquid interfaces are studied. Comparison of Figs. 4(a) and 7(a) shows that at $\Delta_{\mathrm{o}}^{\mathrm{w}} \phi=0$, the potential $\phi^{2}$ in the potential independent model is more negative than that in the Langmuir model. This indicates that in the potential independent model a larger potential drop will be developed in the aqueous phase. Therefore, more adsorbed species will be present at the interface.

According to Eq. (17), the relative surface coverage associated with a specific bulk concentration of the adsorbate is determined by two different exponential terms. The first term is related to the potential independent adsorption, which is a function of the standard Gibbs energy of adsorption. The second term is responsible for the potential dependence of the surface concentration. The respective contributions of these terms to the relative coverage is controlled by the potential difference. Fig. 8 displays the relative coverage in the presence of the adsorption of an ion of charge $z_{\mathrm{b}}=-1$ at the interface. The relative coverage is very low at rather positive potentials, where $\phi^{2}-\phi^{\mathrm{w}}>0$ and the potential independent adsorption is dominant. The coverage slowly increases as the potential difference becomes negative, as manifested by the appearance of a hump in the potential range of $0-0.2 \mathrm{~V}$. In this region, the coverage is controlled by the convolution of two types of adsorp-

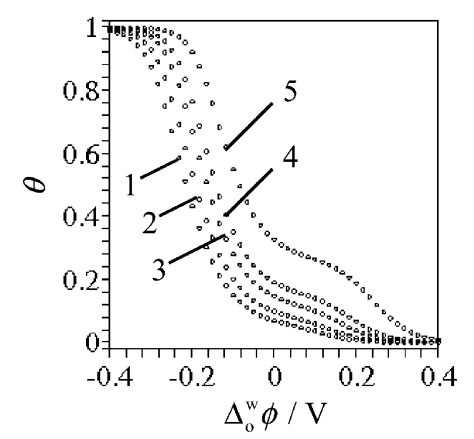

Fig. 8. The effect of $c_{z}$ on the relative surface coverage for the Langmuir model. The parameters are the same as in Fig. 4(a). tion. At more negative potential differences, the potential dependent adsorption becomes dominant and the coverage increases steeply before reaching saturation.

The interfacial charge density and differential capacitance, as calculated with the isotherm in Eq. (17), are plotted in Fig. 9. Again, the minimum of the differential capacitance curve shifts to positive values of $\Delta_{\mathrm{o}}^{\mathrm{w}} \phi$ with increasing bulk concentration of the adsorbate. However, the charge density and differential capacitance curves coincide well at positive potential differences regardless of the concentration of adsorbate, indicating complete desorption of the species at the interface. These features are commonly observed experimentally on the adsorption of anionic species from the aqueous phase to the interface [43-45]. The overlap of the charge density curves, as well as of the differential capacitance curves, at negative potential differences is associated with the saturation of the adsorption at the interface. When the adsorbate reaches full coverage at a certain concentration or potential, no more species will be accepted at the interface. The present model does not account for multilayer assemblies with variations of the Gibbs energy of adsorption.

The results in Fig. 9(b) should be compared with the capacitance curves obtained with the potential independent isotherm (Fig. 6(b)). An important point is that, for equivalent concentrations, the increase in capacitance occurs at more negative potentials in the case of the Langmuir isotherm. At $\Delta_{\mathrm{o}}^{\mathrm{w}} \phi=0$ the capacitance is lower than that in the potential independent model. In both cases, although the inner potential is the same in each phase, the potential $\phi^{2}$ at the interface is negative due
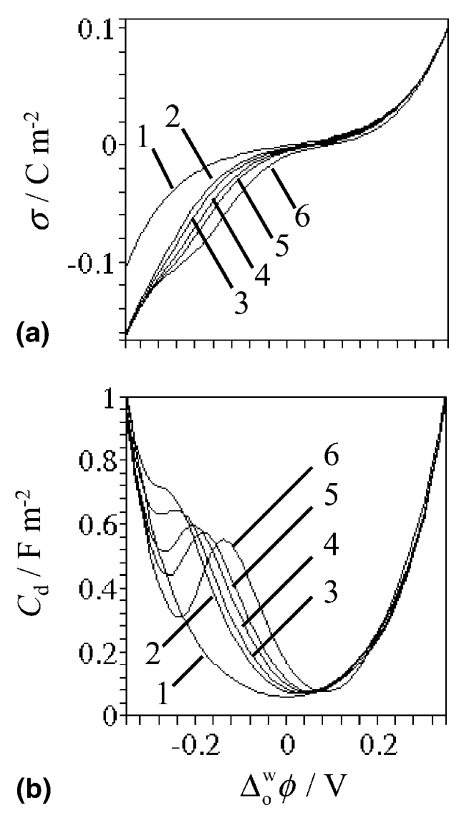

Fig. 9. Charge density (a) and differential capacitance (b) for the Langmuir model at various values of $c_{z}$. The parameters are same as in Fig. 4(a). 
to the adsorption of negatively charged species. Hence the second exponential term in Eq. (17) takes values between 0 and 1 and the surface coverage is decreased. This emphasizes the importance of evaluating the potential profiles at the interface before interpreting capacitance data, as the modification in the electric structure of the interface significantly affects the shape of capacitance-potential curve.

\subsection{Frumkin isotherm}

The Langmuir isotherm represents an ideal case where the intermolecular interactions between adsorbed species are neglected. However, these interactions are expected to play an important role in the adsorption process, especially at high surface coverage. Higgins and Corn [48] have shown that in some cases, the adsorbed species can enhance the incoming adsorption with increasing interfacial coverage. In other cases, repulsion between adsorbates can hinder the adsorption [46]. These phenomena can be conveniently rationalized in terms of the Frumkin isotherm, in which an intermolecular interaction factor is taken into account. The expression for this model is written in the general form

$\ln \left(\frac{\theta}{1-\theta}\right)=\ln \left(\frac{a_{z}}{a_{\mathrm{H}_{2} \mathrm{O}}}\right)-\frac{\Delta G_{\mathrm{a}}^{0}}{R T}-\frac{z F}{R T}\left(\phi^{2}-\phi^{\mathrm{w}}\right)-\frac{x \theta}{R T}$,

where $x$ is the interaction factor (with units of $\mathrm{kJ} \mathrm{mol}^{-1}$ ), which represents the intermolecular interaction between the adsorbed species and is negative in the case of attraction and positive in the case of repulsion. The physical meaning of parameter $x$ is equivalent to that of $g^{\prime}$ in [49], and usually takes values between -5 and $5 \mathrm{~kJ} \mathrm{~mol}^{-1}$.

Although we can work out the symbolic function of $\phi^{2}$ as a function of the applied potential difference, we fail to evaluate it numerically over all values of $x$ and $\Delta_{\mathrm{o}}^{\mathrm{w}} \phi$. As shown in Fig. 10(a), a complete evaluation is possible only with positive values of $x$. In the case of $x=-4 \mathrm{~kJ} \mathrm{~mol}^{-1}$, we merely get a discontinuous curve (Fig. 10(a), curve 4). Fig. 10(b) illustrates the charge density with three assigned value of $x$. We can observe that the charge density decreases due to the repulsive interaction between the adsorbed species.

Owing to the discontinuities in Fig. 10(a), it is difficult to make a complete evaluation of the effect of $x$ on the surface coverage. However, the interfacial coverage can be plotted as a function of the interaction factor and potential difference by taking as an approximation that the variations in $\phi^{2}$ introduced by the interaction between adsorbates can be neglected. Hence, $\phi^{2}$ can be computed using the Langmuir isotherm Eq. (17) and then introduced into Eq. (18) to evaluate the surface coverage. This simplification is reasonable because $x$
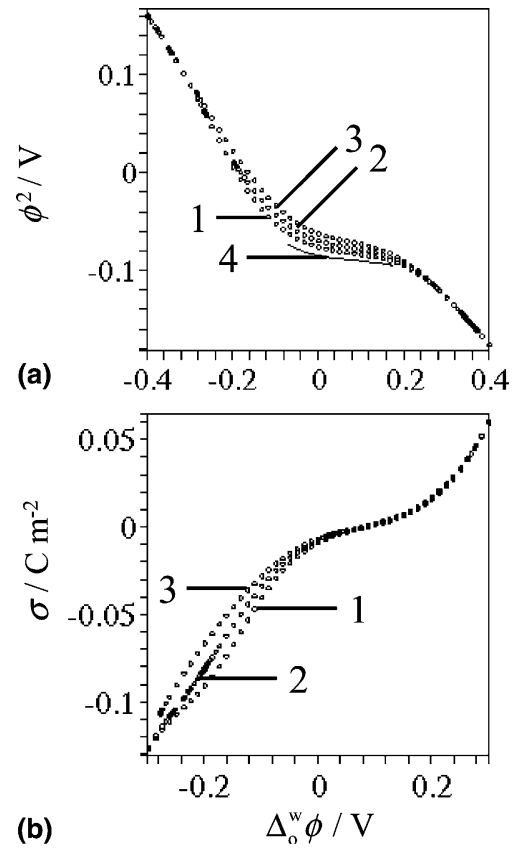

Fig. 10. $\phi^{2}$ (a) and charge density (b) for the Frumkin model at various values of $x ; 0$ (1), 4 (2), 10 (3), and $-4 \mathrm{~kJ} \mathrm{~mol}^{-1}$ (4) and $z_{\mathrm{b}}=-1, \quad \Gamma^{*}=1 \times 10^{-6} \quad \mathrm{~mol} \mathrm{~m}^{-2}, \quad c_{z}=0.050 \quad \mathrm{~mol} \mathrm{~m}^{-3}$, $\Delta G_{\mathrm{a}}^{0}=-40 \mathrm{~kJ} \mathrm{~mol}^{-1}$.

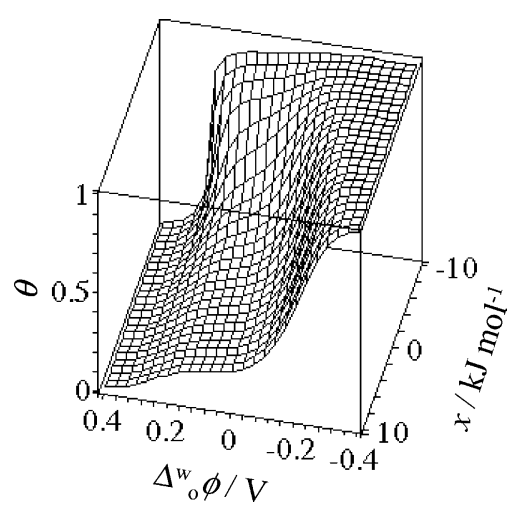

Fig. 11. Three dimensional graph of the relative surface coverage as functions of $\Delta_{\mathrm{o}}^{\mathrm{w}} \phi$ and $x$.

does not induce a large change in $\phi^{2}$ as shown in Fig. 10(a). Fig. 11 is a three-dimensional graph displaying the relative coverage as a function of $x$ and $\Delta_{0}^{\mathrm{w}} \phi$. In the presence of attractive interactions $(x<0)$, the adsorption is enhanced and the saturation is reached at relatively low potential differences. On the other hand, repulsive interactions decrease the surface concentration and shift the saturation potential to more negative values. The intermolecular interaction plays an important role in the extent of the adsorption, which in turn affects the electric structure of the interface.

The capacitance curves obtained for different values of the interaction parameter $x$ are displayed in Fig. 12. The repulsive interaction results in a de- 


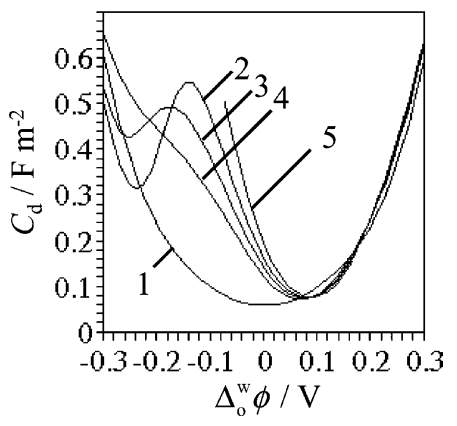

Fig. 12. Differential capacitance for the Frumkin model at $x=0$ (2), 4 (3), 10 (4), and $-4 \mathrm{~kJ} \mathrm{~mol}^{-1}$ (5). Curve 1 is that in the absence of adsorption. Other parameters are the same as in Fig. 5.

crease of the capacitance at negative potentials, as expected from the evolution of the surface coverage in Fig. 11.

\section{Conclusions}

The electric double layer structure of the ITIES is significantly altered by the adsorption of ionic species. On the basis of the common assumptions of the GouyChapman theory, the ionic species are considered as the point charges and the inner layer is reduced to a charged plane. The potential at this plane, $\phi^{2}$, which is resolved using the electro-neutrality condition of the system, determines the magnitudes of the potential drops in the two diffuse layers, $\phi^{2}-\phi^{\mathrm{w}}$ and $\phi^{\mathrm{o}}-\phi^{2}$. Computation of the potential profile across the interface using numerical methods allows the estimation of the charge density and double layer capacity for various adsorption models.

The results clearly show that the potential profile across the interface is affected markedly by the adsorption of ionic species. In a certain potential range, the sign of $\phi^{2}$ is inverted once the adsorbed charge exceeds the excess charge in the diffuse layer regardless of the adsorption model, resulting in a non-monotonic potential distribution with a potential trap at the interface. Obviously, the redistribution of the potential will increase the interfacial charge density and capacity, as well as shift the potential of zero charge. A fundamental question is how this modified potential profile affects the charge transfer at the interface. In terms of the model introduced by Girault and Schiffrin [50] on electron transfer at ITIES, the driving force for the electron transfer includes two work terms associated with the formation of the precursor and successor complexes. The change in the potential distribution alters the local potential drop sensed by the precursor according to Eqs. (10), (17) and (18) in [50]. Hence, the electron transfer rate constant and the transfer coefficient will change in response to the potential redistribution.

\section{Acknowledgements}

We appreciate the financial support of Fonds National Suisse de la Recherche Scientifique.

\section{References}

[1] Z. Samec, Chem. Rev. 88 (1988) 617.

[2] Z. Samec, Surfact. Sci. Ser. 95 (2001) 415.

[3] L. Murtomaki, J.A. Manzanares, S. Mafe, K. Kontturi, Surfact. Sci. Ser. 95 (2001) 533.

[4] C. Gavach, P. Seta, B. D’Epenoux, J. Electroanal. Chem. 83 (1977) 225.

[5] M. Gros, S. Gromb, C. Gavach, J. Electroanal. Chem. 89 (1978) 29.

[6] P. Seta, B. D'Epenoux, C. Gavach, J. Electroanal. Chem. 95 (1979) 191.

[7] Z. Samec, V. Marecek, D. Homolka, J. Electroanal. Chem. 126 (1981) 121.

[8] T. Kakiuchi, M. Senda, Bull. Chem. Soc. Jpn. 56 (1983) 2912.

[9] T. Kakiuchi, M. Senda, Bull. Chem. Soc. Jpn. 56 (1983) 1753.

[10] T. Kakiuchi, M. Senda, Bull. Chem. Soc. Jpn. 56 (1983) 1322.

[11] H.H.J. Girault, D.J. Schiffrin, J. Electroanal. Chem. 170 (1984) 127.

[12] H.H.J. Girault, D.J. Schiffrin, B.D.V. Smith, J. Colloid Interf. Sci. 101 (1984) 257.

[13] H.H.J. Girault, D.J. Schiffrin, J. Electroanal. Chem. 161 (1984) 415.

[14] Z. Samec, V. Marecek, D. Homolka, Faraday Discuss. Chem. Soc. (1984) 197.

[15] Z. Samec, V. Marecek, D. Homolka, J. Electroanal. Chem. 170 (1984) 383.

[16] H.H. Girault, D.J. Schiffrin, J. Electroanal. Chem. 150 (1983) 43.

[17] L.I. Daikhin, M. Urbakh, J. Electroanal. Chem. 560 (2003) 59.

[18] D.S. Walker, M.G. Brown, C.L. McFearin, G.L. Richmond, J. Phys. Chem. B 108 (2004) 2111.

[19] K.J. Schweighofer, I. Benjamin, J. Electroanal. Chem. 391 (1995) 1.

[20] K.J. Schweighofer, I. Benjamin, J. Phys. Chem. 99 (1995) 9974.

[21] I. Benjamin, Annu. Rev. Phys. Chem. 48 (1997) 407.

[22] P.A. Fernandes, M.N.D.S. Cordeiro, J.A.N.F. Gomes, J. Phys. Chem. B 103 (1999) 6290.

[23] W. Schmickler, Surfact. Sci. Ser. 95 (2001) 153.

[24] S. Frank, W. Schmickler, J. Electroanal. Chem. 500 (2001) 491.

[25] S. Frank, W. Schmickler, J. Electroanal. Chem. 564 (2004) 239.

[26] T. Huber, O. Pecina, W. Schmickler, J. Electroanal. Chem. 467 (1999) 203.

[27] C.M. Pereira, W. Schmickler, F. Silva, M.J. Sousa, J. Electroanal. Chem. 436 (1997) 9.

[28] Y. Cheng, V.J. Cunnane, D.J. Schiffrin, L. Murtomaki, K. Kontturi, J. Chem. Soc., Faraday Trans. 87 (1991) 1665.

[29] C.M. Pereira, F. Silva, M.J. Sousa, K. Kontturi, L. Murtomaki, J. Electroanal. Chem. 509 (2001) 148.

[30] L.I. Daikhin, A.A. Kornyshev, M. Urbakh, J. Electroanal. Chem. 500 (2001) 461.

[31] L.I. Daikhin, A.A. Kornyshev, M. Urbakh, Electrochim. Acta 45 (1999) 685.

[32] D.M. Mitrinovic, Z. Zhang, S.M. Williams, Z. Huang, M.L. Schlossman, J. Phys. Chem. B 103 (1999) 1779

[33] M.L. Schlossman, Curr. Opin. Colloid Interf. Sci. 7 (2002) 235.

[34] A.M. Tikhonov, D.M. Mitrinovic, M. Li, Z. Huang, M.L. Schlossman, J. Phys. Chem. B 104 (2000) 6336.

[35] J. Strutwolf, A.L. Barker, M. Gonsalves, D.J. Caruana, P.R. Unwin, D.E. Williams, J.R.P. Webster, J. Electroanal. Chem. 483 (2000) 163 . 
[36] I. Tsuyumoto, N. Noguchi, T. Kitamori, T. Sawada, J. Phys. Chem. B 102 (1998) 2684.

[37] D.J. Fermin, Z. Ding, P.F. Brevet, H.H. Girault, J. Electroanal. Chem. 447 (1998) 125.

[38] T. Kakiuchi, Y. Takasu, M. Senda, Anal. Chem. 64 (1992) 3096.

[39] H. Nagatani, R.A. Iglesias, D.J. Fermin, P.-F. Brevet, H.H. Girault, J. Phys. Chem. B 104 (2000) 6869.

[40] K. Fujiwara, H. Watarai, Langmuir 19 (2003) 2658.

[41] P.-F. Brevet, Surfact. Sci. Ser. 95 (2001) 123.

[42] H.H. Girault, Electrochimie Physique et Analytique, Presses Polytechniques et Universitaires Romandes, 2001.
[43] T. Kakiuchi, M. Kobayashi, M. Senda, Bull. Chem. Soc. Jpn. 60 (1987) 3109.

[44] D.J. Fermin, Z. Ding, H.D. Duong, P.-F. Brevet, H.H. Girault, J. Phys. Chem. B 102 (1998) 10334.

[45] N. Eugster, H. Jensen, D.J. Fermin, H.H. Girault, J. Electroanal. Chem. 560 (2003) 143.

[46] T. Kakiuchi, Surfact. Sci. Ser. 95 (2001) 105.

[47] T. Kakiuchi, J. Electroanal. Chem. 536 (2002) 63.

[48] D.A. Higgins, R.M. Corn, J. Phys. Chem. 97 (1993) 489.

[49] A.J. Bard, L.R. Faulkner, Electrochemical Methods: Fundamentals and Applications, Wiley, New York, 1980.

[50] H.H.J. Girault, D.J. Schiffrin, J. Electroanal. Chem. 244 (1988) 15. 